\title{
ARGUMENTATION MIT TYPOLOGIE IN DER PROTESTANTISCHEN PUBLIZISTIK DES DREISSIGJÄHRIGEN KRIEGES
}

In GOttes vnd deß Heyligen Römischen Reichs Liecht-Butzer, einer proschwedischen Flugschrift aus dem Jahr 1632, wird Gustav II. Adolf von Schweden bezeichnet als "ein Ander Alexander oder Iudas Maccabaeus", der von Gott gesandt worden sei, "sein liebe Kirch in diesen letzten Zeiten nach den göttlichen Weissagungen noch einmahl vor dem End zutrösten/ vnd das Päpstische AntiChristliche Reich zu demütigen".' Der schwedische König ist nicht die einzige historische Persönlichkeit des Dreißigjährigen Krieges, deren militärisches und politisches Handeln in Bezug gesetzt wird zu biblischen und außerbiblischen Figuren der Antike. Bereits 1620 rühmt Martin Opitz in seiner oratio ad serenissimvm ac potentissimvm principem fridericvm regem bohemiae Friedrich $\mathrm{V}$. von der Pfalz als "alter Trajanus" und "alter Augustus", der sich wie seine Vorgänger der Gerechtigkeit verpflichtet habe, ${ }^{2}$ und knapp zwanzig Jahre später erscheint Bernhard von Weimar in einer anläßlich seiner Beisetzung gehaltenen Trauerpredigt als "der Teutschen Josua", "der Teutschen Gideon", "der Teutschen Simson" und "der

1. GOttes vnd deß Heyligen Römischen Reichs Liecht-Butzer. Das ist: Kurtze Erklärung/ wie das Geist- vnnd Weltliche Liecht im Heyligen Römischen Reich/ nämblich die Augspurgische Confession vnnd Religion-Fried/ von den Papisten wollen versteckt vnd gelöscht werden/ vnnd was Gestalt diese Liechter von Jhr Königlichen Majestät in Schweden wider herfür gezogen vnd gebutzet worden/ daß sie dem gantzen Reich zu gutem wider hell vnnd klar leuchten. Allen Vnpassionirten Teutschen Hertzen zur Gedächtnus vnnd Nachrichtung. Jm Jahr/1632. S. 21.

2. "Alter Trajanus es; nemo a vultu tuo discedit tristior: nemo qui justitiam tuam sentit, queritur de saevitia. Imò alter Augustus es, quem dare poenas apparebat cum exigeret" A3v. 
Teutschen Judas [Makkabäus]". ${ }^{3}$ Es handelt sich hier um ein Muster, das bereits im Mittelalter dazu dienen konnte, historische Aktualität zu beschreiben und zu deuten. So stellt sich Karl der Große in die Nachfolge Davids, ${ }^{4}$ und Jahrhunderte später dient die Idee des Davidkönigtums dem staufischen Kaiser Friedrich II. dazu, den Kreuzzug von 1228/29 und die in diesem Zusammenhang erfolgte Einnahme Jerusalems zu begründen und einer religiösen Deutung verfügbar zu machen. ${ }^{5}$ Gemeinsam ist den hier zitierten Beispielen, daß der Vergleich mit Figuren und Situationen aus der Vergangenheit nicht nur eine politisch-militärische, sondern auch und vor allem eine ethisch-religiöse Einordnung der Gegenwart leistet. Personen und Ereignisse werden dargestellt als Protagonisten und Handlungsmomente einer historischen Entwicklung, deren Verlauf göttlichem Willen unterliegt, sie werden typologisch gedeutet. Typologie als bibelhermeneutische Methode hat früh Eingang in den außerbiblischen Bereich gefunden. In Analogie zu dem von zahlreichen Exegeten geübten Verfahren, heilsgeschichtlich bedeutsame Figuren und Ereignisse im Neuen Testament als Erfüllung von im Alten Testament Vorgebildetem zu interpretieren, wird beispielsweise in hagiographischer und panegyrischer Literatur ein typologischer Bezug zwischen einem meist alttestamentlichen Faktum und dessen Vollendung in einem späteren Moment des Geschichtsablaufs postuliert. ${ }^{6}$ Was Typologie leistet, ist demnach

3. Christliche Trawr-Predigt/ Vber den hochbetrawrlichen tödlichen Fall Deß Durchleuchtigsten/ Hochgebornen Fürsten vnd Herrn/ Herrn Bernharden/ Hertzogs zu Sachsen/ [...] Welcher den 8. Julij dieses 1639. Jahrs/ [...] entschlaffen/ Vnd Den 19. diß Monats zu Breisach im Münster [...] beygesetzet worden. [...] Durch danielem rückerum, [...], Aijr.

4. Michael Borgolte: Papst Leo III., Karl der Große und der FilioqueStreit von Jerusalem. In: Byzantina 10(1980), S.401-427, gibt auf S. 403, Anm. 1 einen knappen Überblick über die Forschungsliteratur, die sich mit dem davidischen Königtum Karls des Großen beschäftigt und Belege aufführt für die typologische Legitimation des fränkischen Herrscherhauses.

5. Hans Martin Schaller: Endzeit-Erwartung und Antichrist-Vorstellungen in der Politik des 13. Jahrhunderts. In: Ideologie und Herrschaft im Mittelalter. Hrsg. von Max Kerner. Darmstadt 1982 (= Wege der Forschung 530), S. 303-331.

6. Der diesem Aufsatz zugrunde liegende Typologiebegriff folgt weitgehend den Ausführungen Friedrich Ohlys (Friedrich Ohly: Synagoge und Ecclesia. Typologisches in mittelalterlicher Dichtung. In: Ders.: Schriften zur 
eine heilsgeschichtliche Einordnung: nicht so sehr die physische, intellektuelle oder moralische Qualität einer Persönlichkeit, sondern deren Funktion im göttlich determinierten Ablauf der Zeit wird erhellt. Typologischem Denken liegt ein Geschichtsverständnis zugrunde, das den historischen Ablauf begreift als lineare Entwicklung auf ein religiös definiertes Ziel hin. Geschichte beginnt mit der Erschaffung der Welt, erlebt ihren Angelpunkt in Christus und endet mit dem Jüngsten Gericht. Das Heilswerk Christi markiert eine grundsätzliche Wende und teilt die Heilsgeschichte in zwei voneinander unterscheidbare Stufen, die Zeit vor Christus als Zeit der Verheißung und die Zeit nach Christus als Zeit der Erfüllung. Altes und Neues stehen so in einem Verhältnis der Steigerung, Vergangenes erscheint angesichts einer Gegenwart, in der der gōttliche Heilsplan seine Vollendung findet, als Überwundenes.

Die Beliebtheit dieses Denkmodells ist nicht zufällig. Über Jahrhunderte und in unterschiedlichen Gattungen hat es dazu gedient, Erklärungsmuster für historisches Geschehen anzubieten und das Handeln von Persönlichkeiten aus dem kirchlichen und politischen Bereich zu legitimieren. Das Luthertum, das trotz wiederholter Absagen an die allegorische Schriftauslegung der Bibel keinen endgültigen Bruch mit der katholischen exegetischen Praxis vollzogen hatte, greift im 16. und 17. Jahrhundert typologische Argumentation auf und benutzt sie als wirksame Waffe in der konfessionellen Auseinandersetzung. ${ }^{7}$ Wie und mit welcher Funktion Typo-

mittelalterlichen Bedeutungsforschung. Darmstadt 1977, S. 312-337, und Friedrich Ohly: Typologie als Denkform der Geschichtsbetrachtung. In: Typologie. Internationale Beiträge zur Poetik 2. Hrsg. von Volker Bohn. Frankfurt/M. 1988, S. 22-63). Typologie wird dort definiert als Ordnungssystem, in dem zeitlich Getrenntes aufeinander bezogen wird. Konstitutiv für den Bezug zwischen Alt und Neu, Typus und Antitypus, sind zum einen die Faktizität der Vergleichsmomente - die miteinander in Verbindung gesetzten Figuren und Begebenheiten werden der Geschichte entnommen - und zum andern der qualitative Sprung. Der Antitypus erscheint so als Vollendung eines früher Vorgebildeten auf einer neuen heilsgeschichtlichen Stufe.

7. Heimo Reinitzer: Zur Herkunft und zum Gebrauch der Allegorie im "Biblisch Thierbuch" des Hermann Heinrich Frey. Ein Beitrag zur Tradition evangelisch-lutherischer Schriftauffassung. In: Formen und Funktionen der Allegorie. Symposion Wolfenbüttel 1978. Hrsg. von Walter Haug. Stuttgart 1979 (= Germanistische Symposien-Berichtsbände 3), S. 370-387, weist nach, daß Luthers Kritik an der allegorischen Auslegung biblischer Texte die 
logie in der politisch-konfessionellen Propaganda protestantischer Publizisten des Dreißigjährigen Krieges Anwendung findet, soll im folgenden erläutert werden.

Dem eingangs zitierten Text läßt sich beispielhaft entnehmen, was die typologische Deutung einer historischen Persönlichkeit zu leisten vermag. Der Vergleich Gustav Adolfs mit Alexander dem Großen und dem jüdischen Freiheitskämpfer Judas Makkabäus verweist zunächst auf die militärischen Qualitäten des schwedischen Königs. Sowohl Alexander der Große als auch Judas Makkabäus gelten im 17. Jahrhundert als vorbildliche Heerführer, die sich durch Begabung und Mut gegen ihre Feinde durchzusetzen vermochten. Sie gehören zu den Neun Helden, einer Konstellation von Figuren aus heidnischer Antike, Judentum und christlichem Mittelalter, ${ }^{8}$ die im Barock wiederholt herangezogen wird, um Exponenten zeitgenössischer Politik zu charakterisieren. Gustav Adolf ist jedoch nicht nur ein überragender Heerführer, er handelt, anders als Alexander, bewußt in göttlichem Auftrag. Auch Judas Makkabäus versteht seinen Kampf für die religiöse und politische Selbstbestimmung des jüdischen Volkes als gottbefohlenes Tun, ein Tun allerdings, das vor dem Kommen des Messias und damit auf einer überwundenen Stufe stattfindet. Erst in dem schwedischen König als idealem u n d christlichem Held erfüllt sich der göttliche Heilsplan, der den endgültigen Triumph der ecclesia Christi und den Untergang des Antichrist vorsieht. Der alttestamentliche Konflikt zwischen Juden und nichtjüdischen Völkern wandelt sich nach Christus zur eschatologischen Auseinandersetzung zwischen wahrer Kirche und den antichristlichen Mächten des Bösen, die von den Protestanten in der Nachfolge Luthers mit dem Papsttum identifiziert werden. Angesichts der Notwendigkeit des Kampfes gegen den Antichrist kann die Intervention Gustav Adolfs in den Dreißigjährigen Krieg als

Verwendung allegorischer Deutungsweisen auch bei protestantischen Autoren nicht verhindert hat. Der Rückgriff auf tradierte hermeneutische Grundlagen bot argumentative Möglichkeiten, die gerade die evangelischen Propagandisten geschickt zu nutzen wußten.

8. Bei den antiken, biblischen und christlichen Helden handelt es sich um Hektor, Alexander, Julius Cäsar, Josua, David, Judas Makkabäus, Karl den Großen, König Artus und Gottfried von Bouillon. Zu Ursprung und Verbreitung dieses konventionellen Heldenkanons vgl. Horst Schroeder: Der Topos der Nine Worthies in Literatur und bildender Kunst. Göttingen 1971. 
völlig legitimes Verhalten dargestellt werden, das nicht nur dazu dient, den protestantischen Ständen im Reich eine stärkere militärische und politische Position zu verschaffen, sondern das zugleich den wahren Glauben und die wahre Kirche vor dem Zugriff des Bösen und damit vor dem religiösen Verderben rettet. Die umfassende Rechtfertigung des schwedischen Feldzugs geht Hand in Hand mit einer klaren Verurteilung des Gegners. Die kaiserlichkatholische Partei ist nach den schwedischen Erfolgen Ende 1631 nicht nur militärisch, sondern auch moralisch unterlegen. Da sie sich Gott widersetzt, muß ihr Kampf scheitern, was sie erwartet, ist der physische und religiöse Niedergang.

Folgenreicher als die heilsgeschichtliche Einordnung bedeutender protestantischer Persönlichkeiten war für die politisch-konfessionelle Polemik von lutherischer Seite die bereits angedeutete typologische Deutung der Evangelischen als wahre ecclesia Christi. Der Anspruch der Lutheraner, die einzig legitime Kirche zu sein, stellte die protestantischen Apologeten vor nicht geringe Probleme, bestand doch in Form der katholischen Kirche bereits eine Glaubensgemeinschaft, die sich als typologische Vollendung der alttestamentlichen synagoga verstand. Die evangelischen Publizisten standen vor der Aufgabe, einerseits zu begründen, weshalb die katholische Kirche nicht als rechtmäßige Nachfolgerin der jüdischen Synagoge gelten konnte, und andrerseits die Richtigkeit der Behauptung zu belegen, allein die lutherische Kirche sei die wahre ecclesia. Auf subtile Weise versucht dies der Autor von Das wunder-herrliche Kriegsvnd Siegs-Panier (vgl. Abbildung), eines Flugblatts, das kurz nach dem Sieg der schwedisch-sächsischen Truppen bei Breitenfeld im September 1631 erschienen sein muß. ${ }^{9}$ Der Druck stammt aus der Offizin Gregor Ritzschs, in der zwischen 1630 und 1635 ein be-

9. Beim Autor von Das wunder-herrliche Kriegs- vnd Siegs-Panier könnte es sich um Martin Rinckhart (1586-1649) handeln. Den auf dem Flugblatt abgedruckten Initialen läßt sich entnehmen, daß der Autor den Magistertitel erworben hatte und sich P[oeta] L[aureatus] nennen durfte. Beides trifft auf Rinckhart zu: Der Theologe war nach dem Erwerb des Magister philosophiae von 1617 bis zu seinem Tod als Archidiakon in Eilenburg (Meissen) tătig. Er ist wiederholt als Liederdichter in Erscheinung getreten und wurde für sein poetisches Werk 1613 mit dem Dichterlorbeer gekrönt (vgl. DBA 386-404). Seine engen Beziehungen zu Leipzig könnten ihn veranlaßt haben, die Schlacht bei Breitenfeld in einer prosächsischen Publikation zu verherrlichen. 


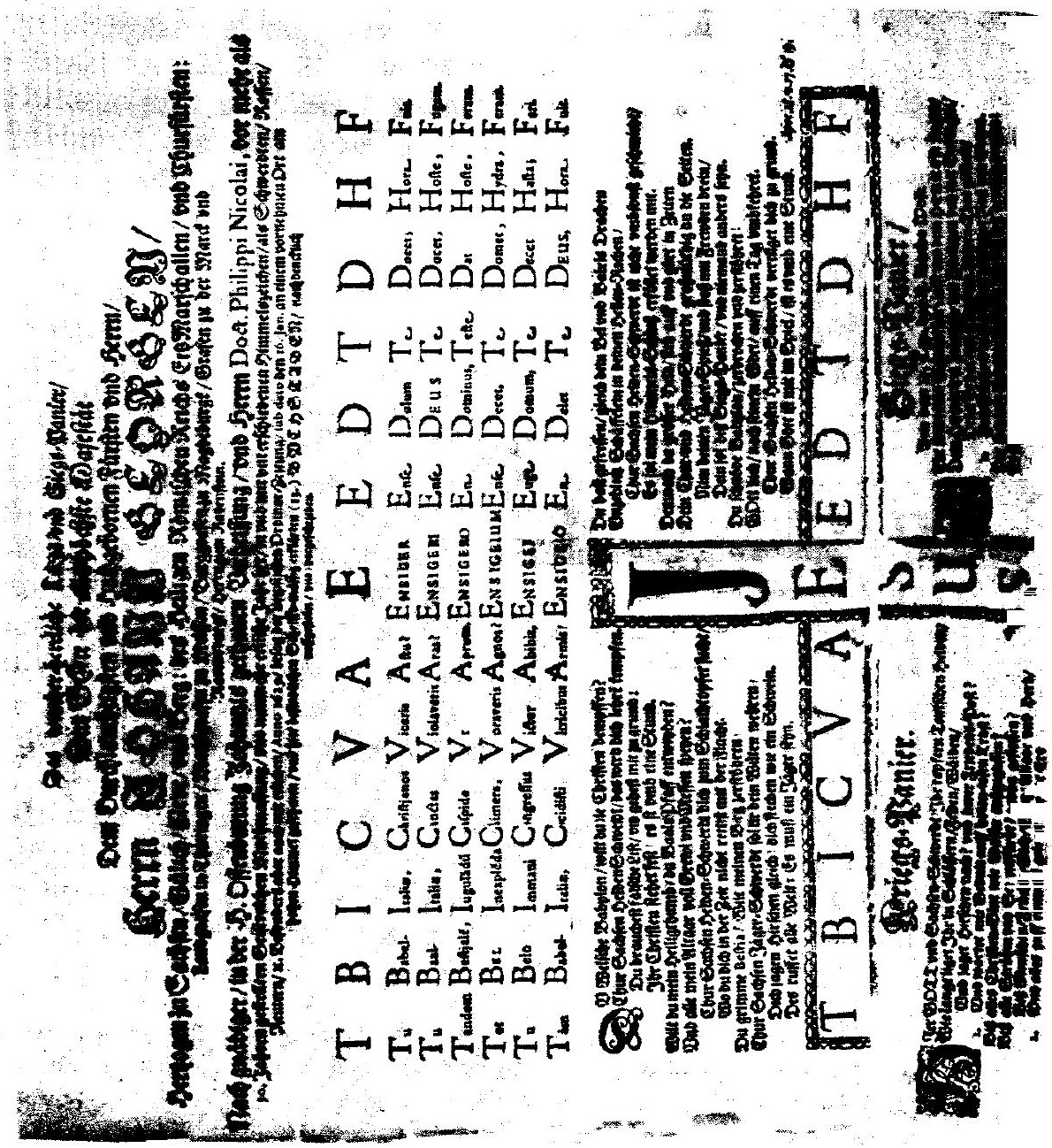




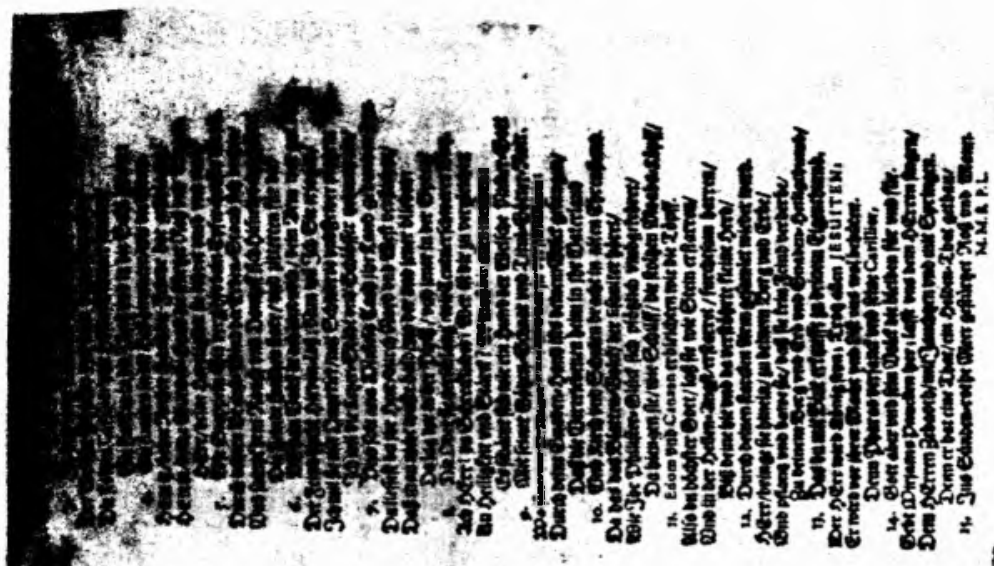

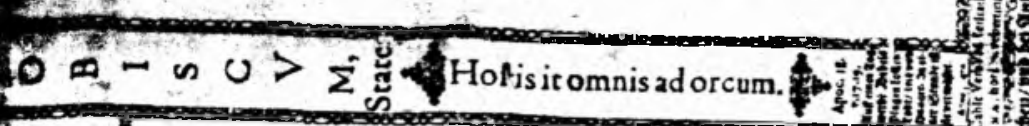

A.

1)

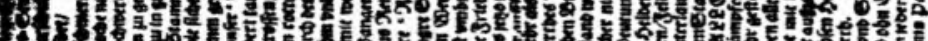

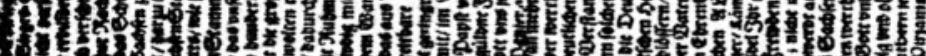

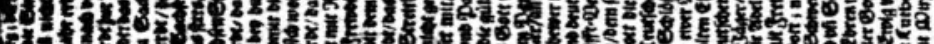
1) Hin

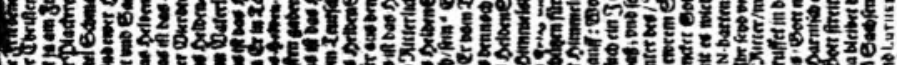
ॠH

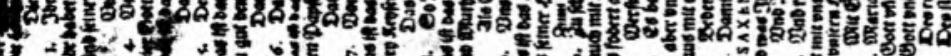

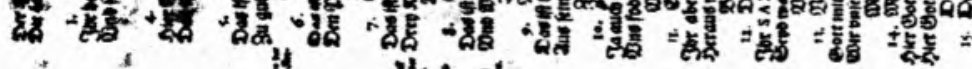

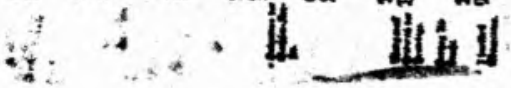


trächtlicher Teil der von offizieller Seite in Auftrag gegebenen sächsischen Publikationen hergestellt wurde. Das wunder-herrliche Kriegs- vnd Siegs-Panier vertritt denn auch eine deutlich kursächsische Position, der schwedische König, ohne dessen Armee die sächsischen Soldaten dem Gegner kaum gewachsen gewesen wären, tritt nicht in Erscheinung.

Zentrales Gestaltungselement ist ein Kreuz, das das Blatt vertikal und horizontal unterteilt. Anstelle der Bezeichnung INRI für Christus ist über dem Kreuz eine Verdammung des römischen Babylon eingefügt, deren anaphorisch gereihte lateinische Vokabeln mit 13 Initialen beginnen, die 1629 am Himmel erschienen sein sollen. Auf der linken Seite des Kreuzes wird im Kriegs-Panier zum Kampf aufgerufen, das Siegs-Panier auf der rechten Seite des Kreuzes aktualisiert den Lobgesang Mirjams aus 2.Mose 15, 1-21: Die Strophen 1 bis 4 beschreiben die Rettung der Israeliten, die Strophen 5 bis 9 berichten über den Verlauf der Schlacht bei Breitenfeld. Durch geographische Bestimmungen wie "Pleiß", "Sahl", "Sprey", Oder und Elbe erfolgt die Lokalisierung. Die Beschreibung einer dichten Staub- und Rauchwolke, die im Heer Verwirrung stiftete, ist historisch belegt. ${ }^{10}$ Wie werden nun biblische Vorlage und aktueller Bericht in Verbindung gebracht? Daß die Wiedergabe des alttestamentlichen Ereignisses mehr als nur exemplarische Funktion hat, zeigt die genauere Betrachtung des ersten Gedichtteils: Das Meer wird als "Sündenroth" bezeichnet, und zu Beginn von Strophe 3 heißt es, Gott habe die Ägypter in "das von Christen-Blut entfärbte SündenMeer" geworfen. Die darauffolgende Zeile spricht vom "Welsche[n] Pharao", und schließlich wird festgehalten, die "stoltzen Cavillier vnd Hosen-Männer" seien in die "Hell" gesunken. Damit offenbart sich im Vergleich eine zusätzliche Dimension: In Analogie zum Untergang des ägyptischen Pharao bedeutet die Niederlage des kaiserlich-katholischen Heeres sowohl den physischen als auch den moralisch-religiösen Zusammenbruch. In der Gegenwart ist jedoch nicht nur eine neue zeitliche, sondern auch eine neue heilsgeschichtliche Stufe erreicht. Der Kampf findet nun im Zeichen des Kreuzes Christi, das das Flugblatt optisch dominiert, und damit im Zeitalter der Gnade statt. Daß es sich

10. Cicely V. Wedgwood: Der Dreißigjährige Krieg. München 1967, S. 261-263. 
dabei um eine eschatologische Auseinandersetzung handelt, wird durch Bezüge zur Apokalypse im Text und in den Inschriften am Fuße des Kreuzes verdeutlicht. Nicht nur um Leben oder Tod, sondern um Heil oder Verdammnis geht es. Die Rollen sind klar verteilt: Die Hölle als Ort der Verdammnis erwartet die katholischen Soldaten, die Aufnahme in das "Gnaden-Heiligthumb" ist den Protestanten als Volk Gottes bestimmt. Der strikte Dualismus, der bereits in den Kreuzinschriften zum Ausdruck kommt - JESUS NOBISCUM lautet die Parole der Protestanten, "Hostis it omnis ad orcum" bleibt den Gegnern vorbehalten - durchzieht den ganzen Text. Das das Kriegs-Panier einleitende "Hier", das in Strophe 14 wieder aufgenommen wird, markiert die Position der Sachsen, die, sich stützend auf das durch Luther vermittelte göttliche Wort, den Beistand des Höchsten in Anspruch nehmen können. Ihnen gegenüber stehen die "JESU-Feind", die kaiserlichen Soldaten, die unter der Führung der Jesuiten das Volk Gottes zu vernichten trachten, wie einst die Philister, Moabiter, Edomiter und Kanaaniter. Daß Letztere nicht mit, sondern gegen Gott kämpfen, ergibt sich aus ihrem verfehlten Glauben, der in Anlehnung an 1. Könige 18, 2040 als Götzendienst dargestellt wird. ${ }^{11}$ Der Abfall von Gott und damit verbunden der Verlust des Erstgeburtsrechts, der in der Bezeichnung "Esauiter" für Jesuiten angedeutet wird, stehen am Anfang einer Entwicklung der Papstkirche, die die katholische Glaubensgemeinschaft in der endzeitlichen Auseinandersetzung als Verkörperung des antichristlichen Zerstörungswillens auftreten läßt. Demgegenüber erscheinen die Protestanten als "verstöhrte kleine Herd" der Rechtgläubigen, die trotz aller Anfechtungen im Bewußtsein agiert, Erbe der göttlichen Verheißung zu sein. Altes Testament und Johannesapokalypse sowie aktuelles Geschehen werden in Das wunder-herrliche Kriegs- vnd Siegs-Panier zu einem heilsgeschichtlichen Konstrukt verdichtet, das die Funktion hat, die religiöse und politische Haltung der Lutheraner zu rechtfertigen. Die Rechtmäßigkeit des evangelischen Glaubens gründet sich in "Gott und Luthers Wort" und manifestiert sich im Triumph über die Feinde; die Notwendigkeit des militärischen Kampfes gegen die kaiserlichen Truppen ergibt sich aus dem eschatologischen Charak-

11. "Wilt du mein Heiligthumb/ du Baals-Pfaff entweyhen? Vnd alle mein Altaar voll Grewl vnd Messen speyen?" 
ter der Auseinandersetzung, die sich als Konfrontation von ecclesia und Antichrist erweist. Dabei kommt der Typologie eine zentrale Rolle zu: Sie verweist gleichzeitig auf die Kontinuität des sich in der Geschichte erfüllendein göttlichen Heilsplans und die Bedeutsamkeit des historischen Moments. Bei aller Ähnlichkeit der Begebenheiten aus der Antike hat das aktuelle Geschehen eine andere Dimension, da es einer neuen heilsgeschichtlichen Stufe angehört. Typologische Geschichtsdeutung erkennt nicht einfach Strukturanalogien zwischen Gegenwart und Vergangenheit, sie liefert, wie bereits zu zeigen versucht wurde, ein Erklärungsmodell für die Ursachen historischer Phänomene. Die Schlacht bei Breitenfeld, die Das wunder-herrliche Kriegs- vnd Siegs-Panier besingt, kann nur so und nicht anders ausgehen, da über Sieg und Niederlage nicht die militärische Potenz der beteiligten Armeen, sondern allein der göttliche Wille entscheidet. Der protestantische Kampf bedeutet nichts anderes als ein Sich-in-Einklang-bringen mit eben diesem Willen.

Die hier beispielhaft vorgeführte religiöse Interpretation historischer Ereignisse der Jahre 1631 und 1632 ist kein Spezifikum protestantischer Publizistik. Analoge Argumentationsmuster finden sich auch im konfessionellen Schriftum katholischer Herkunft. Die größtenteils von Jesuiten verfaßten antilutherischen Flugblätter und Flugschriften bieten eine heilsgeschichtliche Einordnung politischer Aktualität, die nicht wesentlich von derjenigen ihrer protestantischen Kontrahenten abweicht, die Rollen im konfessionellen Konflikt jedoch anders verteilt: Die Römische Kirche tritt hier als Trägerin der Verheißung auf, deren Gegner erscheinen als dem Gericht Gottes verfallene Ketzer. Ein Vergleich zwischen protestantischer und katholischer Publizistik offenbart, in welchem Maße ein in der theologischen Literatur entwickeltes und über kompilatorische Werke wie Exempelbücher, Florilegien, Lexika verbreitetes Instrumentarium für die Produzenten politisch-konfessioneller Polemik verbindlich ist. Die zumeist theologisch geschulten Autoren religiös argumentierender Propaganda greifen unabhängig von ihrer Konfessionszugehörigkeit auf ein Inventar tradierter Bilder und Deutungsmuster zurück, die allerdings je verschieden auf die Gegenwart übertragen werden. Differenzen ergeben sich demnach nicht primär in bezug auf die Form der die jeweilige Botschaft stützenden Argumente, sondern in bezug auf deren Funktion. Dies läßt sich mit 
Blick auf die in diesem Aufsatz genannten Publikationen präzisieren:

Die heilsgeschichtliche Deutung der Schlacht bei Breitenfeld in Das wunder-herrliche Kriegs- vnd Siegs-Panier hat mehrere Funktionen. Indem die katholische Kirche dargestellt wird als Institution, die durch den Abfall vom Wort Gottes und damit vom wahren Glauben den Anspruch, Volk Gottes zu sein, verspielt hat, kann der Ketzervorwurf zurückgewiesen werden. Das "Welsche Babylon" als apokalyptisches Signum Roms ist es, das den wahren Tempel zu entweihen und die Altäre mit "Grewl vnd Messen" zu bedecken versucht. Die Protestanten hingegen als derjenige Teil der ursprünglich geeinten und im Sinne Christi handelnden Kirche, der am Glauben festgehalten hat, verkörpern nun die ecclesia Christi und sind damit alleinige Träger der Verheißung. Über eine theologische Legitimation der lutherischen Kirche hinaus leistet die religiöse Begründung der politisch-militärischen Entwicklung eine Rechtfertigung des Vorgehens der Protestanten im Dreißigjährigen Krieg. Der militärische Widerstand gegen den Kaiser war für nicht wenige Lutheraner nur schwer vereinbar mit der von Luther geforderten Unterwerfung unter die weltliche Obrigkeit. Neben der Uneinigkeit der Evangelischen im Reich mag dies einer der Hauptgründe für die zögernde Haltung insbesondere Sachsens gewesen sein, wenn es darum ging, kaiserlichen Maßnahmen wie dem Restitutionsedikt von 1629, das für die protestantischen Stände eine ernsthafte Bedrohung darstellte, zu begegnen. Nachdem es 1631 unter dem Druck der militärischen Verhältnisse zu einem Bündnis zwischen Schweden und Sachsen gekommen und damit die Entscheidung für ein offensives Vorgehen gegen die kaiserlichen Truppen gefallen ist, sehen sich die proschwedischen und prosächsischen Publizisten vor die Aufgabe gestellt, die Rechtmäßigkeit der protestantischen Initiative zu belegen. Weit wirkungsreicher als eine juristische Begründung des eigenen Handelns scheint dabei die religiöse Beweisführung gewesen zu sein. Die angeführten Beispiele sind nur besonders eindrückliche Belege für eine fast unüberschaubare Publizistik, die mit den immer gleichen Bildern und Argumenten operiert. ${ }^{12}$ Zen-

12. Silvia Serena Tschopp: Heilsgeschichtliche Deutungsmuster in der Publizistik des Dreißigjährigen Krieges. Pro- und antischwedische Propaganda in Deutschland 1628-1635. Frankfurt/M.[u.a] 1991 (= Mikrokosmos 29). 
trales Anliegen all dieser Flugblätter und Flugschriften ist es, den polemischen Nachweis zu erbringen, daß nicht die katholische Kirche, sondern die lutherische ecclesia Christi als typologische Vollendung der synagoga zu gelten hat, daß die Exponenten der protestantischen Partei als von Gott Beauftragte erfüllen, was im Alten Testament in typologischem Sinne vorgebildet ist. Heilsgeschichte - dies ist die Botschaft der typologisch argumentierenden Propagandaschriften - setzt sich fort in den Protestanten als neuem Volk Israel und vollendet sich damit außerhalb des Katholizismus. Eine derartige Strategie erlaubt es den Lutheranern, die Tradition für sich in Anspruch zu nehmen. Sie lehnen das Vergangene nicht grundsätzlich $a b$, sondern ordnen sich ein in die Kirchengeschichte als "Häuflein der Gerechten", das sich gegen alle Versuchungen behauptet hat und in dem Gott deshalb seine VerheiBung erfüllt. Die Verdeutlichung der heilsgeschichtlichen Funktion zeitgenössischer Persönlichkeiten und Ereignisse dient demnach nicht nur ihrer Legitimation, sondern auch dazu, den Protestanten die historische Verankerung und Kontinuität zu verleihen, die die römische Kirche ihnen abspricht. Die Verwendung überlieferter hermeneutischer Methoden in protestantischer Propaganda läßt sich demnach nicht nur mit dem Beharrungsvermögen von Denkschemata bei Produzenten und Rezipienten politisch-konfessioneller Publizistik erklären. Sie verweist vielmehr auf eine Strategie, die darauf zielt, im Akt der Aneignung von Tradition zu verdeutlichen, daß das zu Vermittelnde nicht nur inhaltliche, sondern auch formale Dignität besitzt und so gewissermaßen doppelt legitimiert ist. Die Beherrschung der Tradition, das souveräne Verfügen über ein argumentatives Instrumentarium, dessen Wert allgemein anerkannt ist, ermöglichen es, in der konfessionellen Auseinandersetzung diskurswürdig zu bleiben und zugleich die Protestanten als primäre Adressaten der hier untersuchten Publikationen davon zu überzeugen, daß sie ein gültiges, autorisiertes Glaubensfundament ihr eigen nennen können. Der bewußte Einsatz legitimierender Verfahrensweisen in Das wunder-herrliche Kriegs- vnd Siegs-Panier neben Typologie wären Allegorie (der Kampf gegen die katholischen Truppen wird unter anderem dargestellt als Hirschjagd und Schweinehatz), Etymologie (die sächsischen Soldaten werden als "SAXEN-harte Kerl" und "ALLE-Männer" bezeichnet), Berufung auf autoritätsstiftende Quellen (es wird nicht nur auf die Bibel, 
sondern auch auf außerbiblische Quellen wie Philipp Nicolais Commentarii de Regno Christi verwiesen) und Prophetie sowie Prognostik zu nennen - dient, unter kluger Berücksichtigung des Erwartungshorizonts der Adressaten, dazu, der insbesondere von sächsischen Theologen vorangetriebenen Institutionalisierung der lutherischen Kirche in der ersten Hälfte des 17. Jahrhunderts Vorschub zu leisten und eine Legitimationsbasis zu schaffen, die den Angriffen der konfessionellen Gegner standhält. 